\title{
Cryptic genetic variation in parasitic protozoa
}

\begin{abstract}
Historically, protozoa have always been identified and classified on the basis of their morphology. In some groups, the morphology can be very complex and may offer several features that are diagnostic for particular species even by light microscopy. However, partly because of our reliance on this method for identification we have been led into a trap: namely, making the assumption that if we cannot tell two organisms apart by morphology then they must belong to the same species. The availability of molecular methods for detecting genetic diversity has forced a re-evaluation of this view. This is especially true of certain groups of parasitic protozoa, in which the morphology is often quite simple. This brief review highlights a few examples of our changing understanding of these organisms and the resulting implications for diagnosis, treatment and epidemiology.
\end{abstract}

\section{Entamoeba histolytica}

Perhaps the best example of morphology having led us astray is that of E. histolytica. This organism is the agent of amoebic dysentery and amoebic liver abscess and is responsible for an estimated 50-100000 deaths/year. The figures quoted in most textbooks state that up to $10 \%$ of the world's population is infected and that $<10 \%$ of those infected develop symptoms. The underlying reason for this low morbidity and mortality started to become clear in the late 1970s with the use of iso-enzyme electrophoresis. This approach showed that E. histolytica isolates could be subdivided into two groups based on their association with symptoms: organisms isolated from people with disease 'pathogenic' E. histolytica - could be distinguished from those present in most cases of asymptomatic infection - 'non-pathogenic' E. histolytica [1]. Subsequently, the iso-enzyme data were complemented by antigenic and DNA differences that all pointed to the existence of two distinct and non-overlapping entities within what was being called E. histolytica. Based on these findings the two groups were re-described as distinct species; the one associated with disease retained the name E. histolytica and the other, which appears never to cause disease, is now known as $E$. dispar [2]. The idea that $E$. dispar existed as a separate species was first proposed 75 years ago, but was rejected at the time, partly because of the absence of morphological differences [3].
The recognition of these two Entamoeba species has significant implications. Almost all diagnosis of infection has been, and is still, based on light microscopy. As a result of the two species being morphologically indistinguishable, the species that was really observed cannot be deduced from records of parasite prevalence in the literature. Unless haematophagous trophozoites were noted as having been observed in the stool sample - a characteristic of $E$. histolytica only - existing data based on microscopic observation alone are useless. Only data on the prevalence of amoebic liver abscess, if correctly diagnosed, are reliable. The true prevalence of $E$. histolytica in asymptomatic individuals is uncertain. However, it is suspected that $>90 \%$ of microscopically identified infections are due to E. dispar and that only a proportion of true E. histolytica infections go on to cause invasive disease. Because of this, the WHO recommendations [4] now state that asymptomatic infections should not be treated unless there is definitive species identification or some other reason to suspect that E. histolytica is present. The only method commercially available for positive identification of the two species is a stool antigen capture ELISA kit [5], although many other molecular techniques have been described.

There are several species of parasitic protozoa in which significant levels of genetic variation are known to exist, but only rarely is the significance understood. In $E$. histolytica there was a clear-cut correlation between genetic markers and pathogenicity that justified the recognition of two distinct species. In other species of protozoa the situation is not so clear. At the moment, only in the case of E. histolytica have species names been proposed and accepted for the subgroups. In part, this is because there are no accepted criteria for defining species in what are usually asexual organisms. However, in some cases the topic is now under discussion.

\section{Cryptosporidium}

C. parvum is the name given to the species that infects human beings. However, it has become clear that two quite different organisms exist within this taxon. As in E. histolytica, iso-enzymes provided the first evidence for two distinct genetic groups and the dichotomy has been reinforced by numerous genetic markers. One 
group of C. parvum is largely or exclusively confined to human infections whereas the other is found mostly in domestic and farm animals, but is also responsible for some human infections [6]. Once again there are no morphological differences, but the two forms do appear to have different transmission cycles, as no organisms with a mixture of genetic markers have been described. The interpretation is that all infections with the 'human' variant must have been acquired from other human beings. The epidemiological implications for transmission are significant but, again, prevalence data collected by microscopy alone are uninformative. Numerous methods for differentiating the two forms exist, but only as research tools. There is no clear-cut evidence for differences in disease potential between the two variants, as each can be found in both symptomatic and asymptomatic infections. The establishment of distinct species names for the two forms is under discussion, but no formal taxonomic proposal has yet been made [6].

\section{Giardia}

Again, all human infections are ascribed to one species - G. intestinalis (also known as G. lamblia or $G$. duodenalis) - and as in C. parvum there is evidence of some zoonotic transmission. There appear to be two major lineages, but there is no clear host specificity in this case [7]. Although the degree of genetic variation is significant, the disease caused by the variants appears to be the same. The genetic diversity is thus of considerable interest to laboratory researchers but has little impact on the outcome of infection or its treatment.

\section{Trypanosoma cruzi}

Genetic diversity in $T$. cruzi was also first studied with iso-enzymes, and DNA markers have subsequently confirmed that there are two major lineages that differ significantly [8]. As yet, there is no obvious link between the lineages and specific types of disease. However, one lineage appears to be associated primarily with sylvatic transmission cycles, while the other is more common in human infections [9]. This is again reminiscent of the situation in C. parvum. Species or subspecies names are being discussed for the two lineages but, because there is some evidence for genetic exchange between them, this is probably not a good idea.

\section{Blastocystis and Dientamoeba}

Cryptic genetic diversity is not confined to pathogens but exists within commensal organisms as well. Other species of Entamoeba that colonise the human gut, such as E. coli, show variation on at least the same scale as that seen in E. histolytica/E. dispar [10]. Because these organisms are not associated with disease this observation is of little practical importance. However, there are other situations where this may not be the case. B. hominis and D. fragilis are two organisms whose link to human disease is a matter of controversy $[11,12]$.

B. hominis is probably the most common human gut protozoan in the world. Prevalence rates of $>50 \%$ are regularly reported in developing countries [11], while D. fragilis appears to be much less common. The uncertainty over their role in disease arises in part from the non-specificity of the symptoms associated with their presence. The clinical picture with these two organisms is similar: they have each been associated with diarrhoea and other relatively mild gastrointestinal complaints. The epidemiological picture is less clear. Surveys have shown the presence of $B$. hominis and $D$. fragilis in many asymptomatic individuals and rates of infection in symptomatic and control groups are often not significantly different. However, in individual cases the parasite is the only identified cause of the symptoms and these often disappear when the organisms are eradicated. One possible explanation for this disparity could be that there are morphologically identical genetic variants within each of these species, only one or some of which are causing symptoms.

Analyses of ribosomal genes have shown that sequence variants do exist in both species. In B. hominis the amount of variation is very large [13]. At least eight genetically distinct forms that fall into three lineages have been detected in human infections so far [13; and unpublished observations]. Two variants have been detected in D. fragilis (J. Johnson and C. G. Clark, unpublished observations). If only one cluster or one variant was causing disease, the clinical and epidemiological observations could be reconciled.

It is not known whether the genetic variation found in $B$. hominis and D. fragilis is significant in the clinical context and it may prove difficult to ascertain. Whether the genetic variation eventually proves irrelevant in these cases or not, they serve to illustrate the fact that much remains unknown about genetic diversity in human parasites. The protozoologist's traditional reliance on morphological identification may have lured us into traps that are only now being recognised.

C. GRAHAM CLARK

Department of Infectious and Tropical Diseases, London School of Hygiene and Tropical Medicine, Keppel Street, London WC1E 7HT (e-mail: graham.clark@1shtm.ac.uk)

\section{References}

1. Sargeaunt PG. The reliability of Entamoeba histolytica zymodemes in clinical diagnosis. Parasitol Today 1987; 3: $40-43$. 
2. Diamond LS, Clark CG. A redescription of Entamoeba histolytica Schaudinn, 1903 (Emended Walker, 1911) separating it from Entamoeba dispar Brumpt, 1925. J Euk Microbiol 1993; 40: 340-344.

3. Brumpt E. Differentiation of the human intestinal amoebae with four-nucleated cysts. Trans R Soc Trop Med Hyg 1928; 22: 101-114 (Discussion pp. 115-124).

4. Anonymous. Entamoeba taxonomy. Bull World Health Organ 1997; 75: 291-292.

5. Haque R, Ali IKM, Akther S, Petri WA. Comparison of PCR, isoenzyme analysis, and antigen detection for diagnosis of Entamoeba histolytica infection. J Clin Microbiol 1998; 36: $449-452$.

6. Morgan UM, Xiao L, Fayer R, Lal AA, Thompson RCA. Variation in Cryptosporidium: towards a taxonomic revision of the genus. Int $J$ Parasitol 1999; 29: 1733-1751.

7. Andrews RH, Monis PT, Ey PL, Mayrhofer G. Comparison of the levels of intra-specific genetic variation within Giardia muris and Giardia intestinalis. Int $J$ Parasitol 1998; 28: $1179-1185$.
8. Briones MRS, Souto RP, Stolf BS, Zingales B. The evolution of two Trypanosoma cruzi subgroups inferred from rRNA genes can be correlated with the interchange of American mammalian faunas in the Cenozoic and has implications to pathogenicity and host specificity. Mol Biochem Parasitol 1999; 104: 219-232.

9. Fernandes O, Souto RP, Castro JA et al. Brazilian isolates of Trypanosoma cruzi from humans and triatomines classified into two lineages using mini-exon and ribosomal RNA sequences. Am J Trop Med Hyg 1998; 58: 807-811.

10. Clark CG, Diamond LS. Intraspecific variation and phylogenetic relationships in the genus Entamoeba as revealed by riboprinting. J Euk Microbiol 1997; 44: 142-154.

11. Stenzel DJ, Boreham PF. Blastocystis hominis revisited. Clin Microbiol Rev 1996; 9: 563-584.

12. Grendon JH, DiGiacomo RF, Frost FJ. Descriptive features of Dientamoeba fragilis infections. J Trop Med Hyg 1995; 98 309-315.

13. Clark CG. Extensive genetic diversity in Blastocystis hominis. Mol Biochem Parasitol 1997; 87: 79-83. 